\title{
Computational archaeology of the Pristionchus pacificus genome reveals evidence of horizontal gene transfers from insects
}

\author{
Christian Rödelsperger and Ralf J Sommer
}

\begin{abstract}
Background: The recent sequencing of nematode genomes has laid the basis for comparative genomics approaches to study the impact of horizontal gene transfer (HGT) on the adaptation to new environments and the evolution of parasitism. In the beetle associated nematode Pristionchus pacificus HGT events were found to involve cellulase genes of microbial origin and Diapausin genes that are known from beetles, but not from other nematodes. The insect-to-nematode horizontal transfer is of special interest given that $P$. pacificus shows a tight association with insects.

Results: In this study we utilized the observation that horizontally transferred genes often exhibit codon usage patterns more similar to that of the donor than that of the acceptor genome. We introduced GC-normalized relative codon frequencies as a measure to detect characteristic features of $P$. pacificus orphan genes that show no homology to other nematode genes. We found that atypical codon usage is particularly prevalent in $P$. pacificus orphans. By comparing codon usage profiles of 71 species, we detected the most significant enrichment in insectlike codon usage profiles. In cross-species comparisons, we identified 509 HGT candidates that show a significantly higher similarity to insect-like profiles than genes with nematode homologs. The most abundant gene family among these genes are non-LTR retrotransposons. Speculating that retrotransposons might have served as carriers of foreign genetic material, we found a significant local clustering tendency of orphan genes in the vicinity of retrotransposons.

Conclusions: Our study combined codon usage bias, phylogenetic analysis, and genomic colocalization into a general picture of the computational archaeology of the P. pacificus genome and suggests that a substantial fraction of the gene repertoire is of insect origin. We propose that the Pristionchus-beetle association has facilitated HGT and discuss potential vectors of these events.
\end{abstract}

\section{Background}

The unique genetic repertoire that allows organisms to adapt to environmental changes and to conquer ecological niches has undergone an enormous evolutionary history. Evolutionary events such as speciation, gene duplication, and loss, accompanied by changes on the single nucleotide level, have generated an overwhelming amount of diversity in all domains of life. In addition to the continuous transfer of genetic material to the subsequent generations by means of inheritance, a number of examples are known where genes are transferred across

\footnotetext{
* Correspondence: ralf.sommer@tuebingen.mpg.de

Department for Evolutionary Biology, Max-Planck Institute for Developmental Biology, Spemannstrasse 37, 72076 Tübingen, Germany
}

species borders. Such cases of horizontal gene transfer (HGT) commonly occur in prokaryotes [1,2]. More recently, a number of HGT events have been reported in nematodes [3-6]. The nematode Pristionchus pacificus has initially been introduced as a satellite system for comparison to developmental processes of Caenorhabditis elegans [7]. P. pacificus has a necromenic lifestyle. In the wild, it exists in the form of dauer larvae in association with scarab beetles [8]. Dauer larvae only resume development and become adult worms after the death of the beetle. Initial analysis of the genome sequence identified seven cellulase genes which are of microbial origin [9]. In contrast to cellulases in plant parasitic nematodes, $P$. pacificus cellulases originate from independent HGT

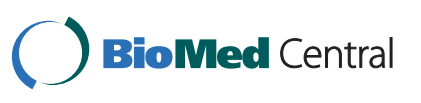

(c) 2011 Rödelsperger and Sommer; licensee BioMed Central Ltd. This is an Open Access article distributed under the terms of the Creative Commons Attribution License (http://creativecommons.org/licenses/by/2.0), which permits unrestricted use, distribution, and reproduction in any medium, provided the original work is properly cited. 
events $[3,9]$. One striking feature of the $P$. pacificus genome is that for more than a third of the 24,231 predicted genes, no homologous sequences exist in any other organism. However, over $50 \%$ of these genes show evidence for expression in the form of expressed sequence tag (EST) data [10]. Thus the origin of these so called 'pioneer genes' remains unclear. One open question is whether these genes are Pristionchus-specific inventions or whether they have been integrated by means of HGT. The lack of homology to any known protein sequence is an ultimate impediment to any kind of phylogenetic analysis. Therefore, alternative approaches for sequence comparisons are preferable to further characterize these genes.

Early after the initial sequencing of the Escherichia coli genome, computational approaches were used to elucidate the molecular archaeology of the E. coli genome [1]. This involved the identification of foreign DNA introduced by HGT. Most of the detection methods for so called "alien" DNA search for sequences that differ from the rest of the genome with respect to a certain feature such as dinucleotide distribution $[11,12]$ or codon usage $[1,13,14]$.

In this study we will compare codon usage profiles of $P$. pacificus genes within and across genomes. Focusing on orphan genes that do not show homology to any other nematode sequence, we use dozens of genomewide profiles for nematodes, bacteria, fungi, insects and plants to show that the $P$. pacificus orphan genes are strongly enriched in insect-like codon usage. In cases where homology data is available, genes with predicted insectlike codon usage show a significant association with HGT candidates defined by cross-species homology. In a more detailed analysis of the common HGT candidates, we investigate the role of retrotransposons in the prospective transfer of genetic material from insects to the $P$. pacificus genome.

\section{Results}

\section{Orphan genes are associated with atypical codon usage}

Every genome has a unique pattern of relative synonymous codon usage (RSCU) (see [15] for review). Alternative synonymous codon usage has been associated with tRNA abundances, translational robustness, mRNA secondary structures and genomic GC content. Consistent with this observation, differences across genomes are greater than differences within genomes. Thus recently introduced genes may be identified when their codon usage bears greater resemblance to the donor than the acceptor genome. As a consequence, methods have been developed to detect alien genes with atypical codon usage profiles relative to the genomewide average [1,13,14].

Foremost, we tested if atypical RSCU profiles of $P$. pacificus could be used to detect genes that presumably originate from species outside of the nematode phylum.
To do so, we determined whether genes with atypical RSCU profiles are enriched in the set of 9217 P. pacificus orphan genes, i.e. genes that do not have a homolog in any other nematode species (see Methods). One confounding factor in the measurement of atypical codon usage is the genomewide variation of GC content; it has been shown that codon usage in nematodes is largely affected by variations in GC content across different species [16]. To assess the impact of GC content on codon usage we calculated the average RSCU profile for $P$. pacificus and tested whether the Euclidean distance between the genomewide profile and the RSCU profiles of individual genes correlate with differences in GC content of the third codon position (GC3). We indeed found good correlations between deviations in RSCU and GC3 content ( $r=0.57$, Pearson, Figure 1A). In addition, we observed a correlation of $r=0.44$ between intronic GC content and GC3 content (Figure $1 B)$, suggesting that intraspecies-specific genomic variations in GC content largely affect variations in RSCU profiles for individual genes.

To decrease the impact of GC content on RSCU values, we introduced GC-normalized RSCU values which drop the assumption that all synonymous codons are equally likely. This also takes into account the GC3 content of the whole gene in the calculation of expected codon frequencies (see Methods). GC normalization substantially decreases the correlation between RSCU differences and differences in GC3 content to $r=0.21$ (Figure 1C). Additional file 1 shows the distribution of GC-normalized RSCU deviation from the genomewide profile. We subsequently tested whether the three deviation measures RSCU, GC-normalized RSCU, and GC3 are associated with $P$. pacificus orphan genes. We ranked all genes according to decreasing values of each deviation measure and calculated the fraction of orphans in the set of most atypical genes, as defined by various cutoffs. Figure 1D shows the resulting cumulative distribution of orphan genes with respect to the three measures. In comparison to the deviation in RSCU and GC3, the GC-normalized RSCU measure shows a strong enrichment (up to two-fold for the first $5 \%)$ of $P$. pacificus orphan genes $(P=0.0002$, one-sample KS-test). Differences in non-normalized RSCU yield an enrichment in orphan genes that is two orders of magnitude less significant $(P=0.02)$. In contrast, deviation in GC3 content alone shows no significant enrichment at all $(P=1)$. These results suggest that a large fraction of $P$. pacificus orphan genes show indeed atypical patterns of synonymous codon usage, a trend that cannot be explained by variation in GC3 content.

\section{Orphan genes show elevated levels of insect-like codon usage}

Next we sought to explore if the atypical codon usage could be used to identify the donor through which these 


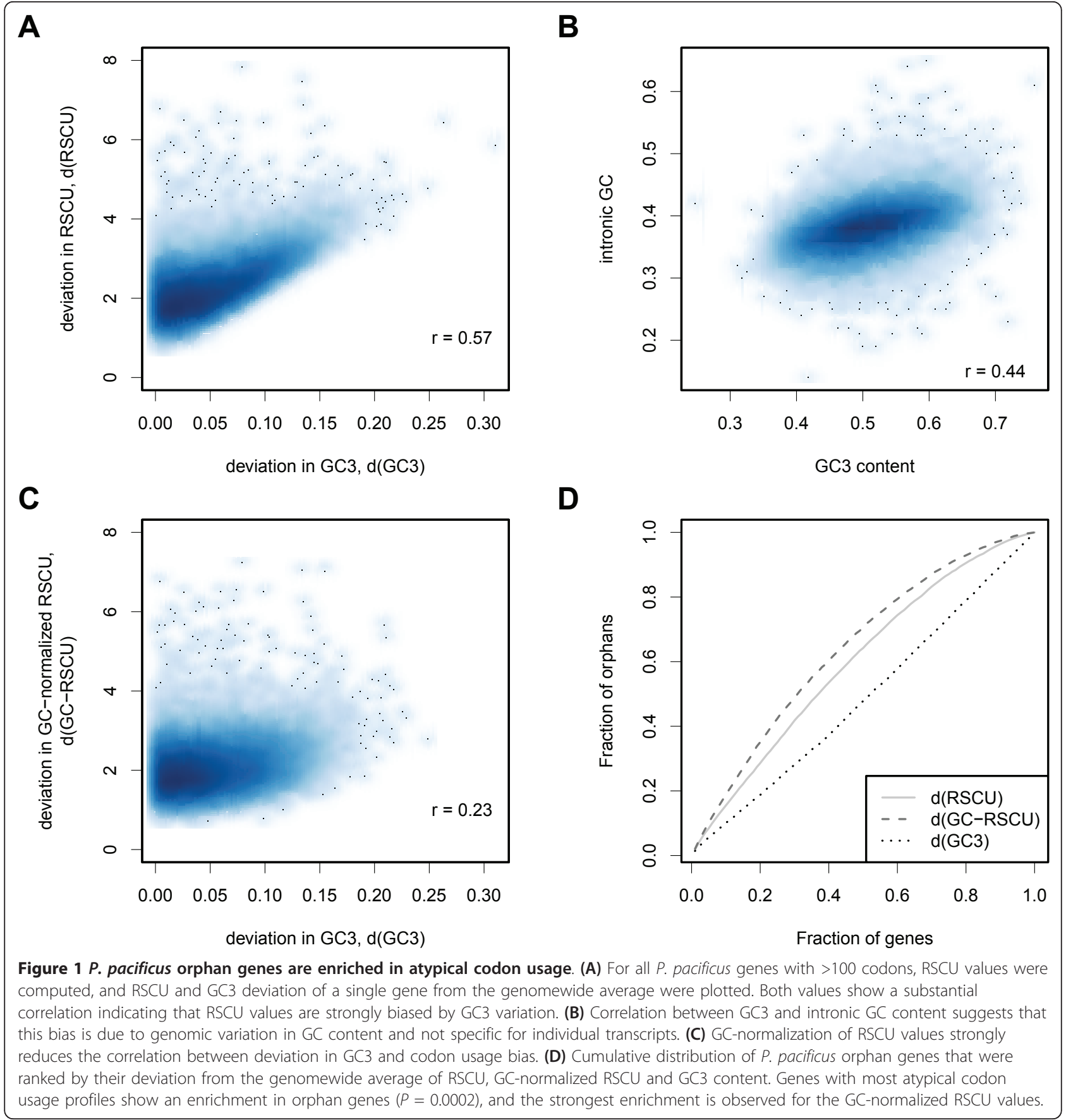

genes entered the $P$. pacificus genome. To this end, we compiled a data set of coding sequences totaling 71 bacterial, fungal, amoebozoa, plant, and insect genomes and tested whether orphan genes show a tendency for enrichment in a particular taxonomic group. First, we calculated genomewide GC-normalized RSCU profiles for each species and assigned individual genes to the species with the closest Euclidean distance in RSCU profiles. This "nearest neighbor" approach was able to predict the correct species in $34.4 \%$ of the cases and the correct taxonomic group in $71.7 \%$ (Table 1). While classification errors exist, they represent a minority of genes and vary across taxonomic groups. For example, it is much more likely that a randomly selected insect gene is mispredicted as a nematode gene $(\sim 22 \%$ misclassifications, Table 1$)$ than vice versa $(\sim 12 \%$ misclassifications, Table 1). Given the correct classification of genes in the majority of cases, the nearest neighbor method is well 
Table 1 Accuracies of taxonomic group predictions based on GC-normalized codon usage profiles

\begin{tabular}{|c|c|c|c|c|c|c|}
\hline Taxonomic group & insect* & bacteria* & amoebozoa* & fungi* & nematode* & plant * \\
\hline Insects & $61.1 \pm 0.7$ & $6.8 \pm 0.5$ & $0.3 \pm 0.1$ & $7 \pm 0.4$ & $22.0 \pm 0.3$ & $2.8 \pm 0.2$ \\
\hline Bacteria & $8.5 \pm 0.2$ & $85.5 \pm 0.4$ & $0 \pm 0$ & $1.9 \pm 0.1$ & $3.6 \pm 0.2$ & $0.5 \pm 0.1$ \\
\hline Amoebozoa & $2.2 \pm 0.6$ & $1.5 \pm 0.3$ & $82.9 \pm 1.2$ & $1.7 \pm 0.7$ & $10.8 \pm 1.7$ & $0.9 \pm 0.6$ \\
\hline Fungi & $10.3 \pm 0.6$ & $1.3 \pm 0.1$ & $0.2 \pm 0.1$ & $71.3 \pm 0.7$ & $9.1 \pm 0.5$ & $7.9 \pm 0.3$ \\
\hline Nematodes & $12.0 \pm 0.4$ & $5.5 \pm 0.2$ & $0.5 \pm 0.1$ & $7.1 \pm 0.2$ & $71.9 \pm 0.6$ & $3.0 \pm 0.2$ \\
\hline Plants & $6.8 \pm 0.8$ & $2.9 \pm 0.4$ & $0.3 \pm 0.1$ & $18.1 \pm 0.6$ & $13.9 \pm 0.9$ & $58.0 \pm 1.2$ \\
\hline
\end{tabular}

Accuracy was measured using ten randomized data sets including equal number of sequences per species (see Methods). For each taxonomic group (rows), we calculated the mean percentage of predictions for all possible classifications, the "*" indicates the predicted taxonomic group (columns). For instance, while $61.1 \%$ of insect genes are correctly recognized as insect sequences based on their codon usage, $22.0 \%$ are mistaken as nematode genes.

suited to suggest the taxonomic group of a potential donor species for HGT events. In addition to the nearest neighbor approach, we also applied a classification method based on random forests [17] and tested this method on GC-normalized RSCU as well as nonnormalized RSCU values and taking into account gene size and GC3 content. However this method did not qualitatively change the results.

Next, we used the nearest neighbor approach to assign all $P$. pacificus genes to taxonomic groups. Figure 2A shows the distribution of taxonomic group classification results. $86.5 \%$ of $P$. pacificus genes are classified as nematode genes. The remaining $13.5 \%$ is distributed among fungi $(6.8 \%)$, insect (4.8\%), plants (1.2\%), and bacteria $(0.6 \%)$. This distribution is most likely influenced by the classification error discussed previously; however, the genomewide distribution may serve as a baseline level for comparison with subsets of genes. If we consider only orphan genes, nematode-like codon usage is strongly depleted. Interestingly, the most significant enrichment for orphan genes is observed in insectlike codon usage $\left(P<10^{-54}\right.$, Fisher's exact test). Specifically, $8.5 \%$ of the analyzed orphan genes are classified as insect genes. When applied to the whole genome, this value represents $54.7 \%$ of all genes that are classified as insect genes and denotes a 1.8 fold enrichment relative to all genes (Figure 2B).

\section{HGT candidates defined by cross-species homology are associated with insect-like codon usage}

Given the strong enrichment of insect-like codon usage in P. pacificus orphan genes, we searched for further

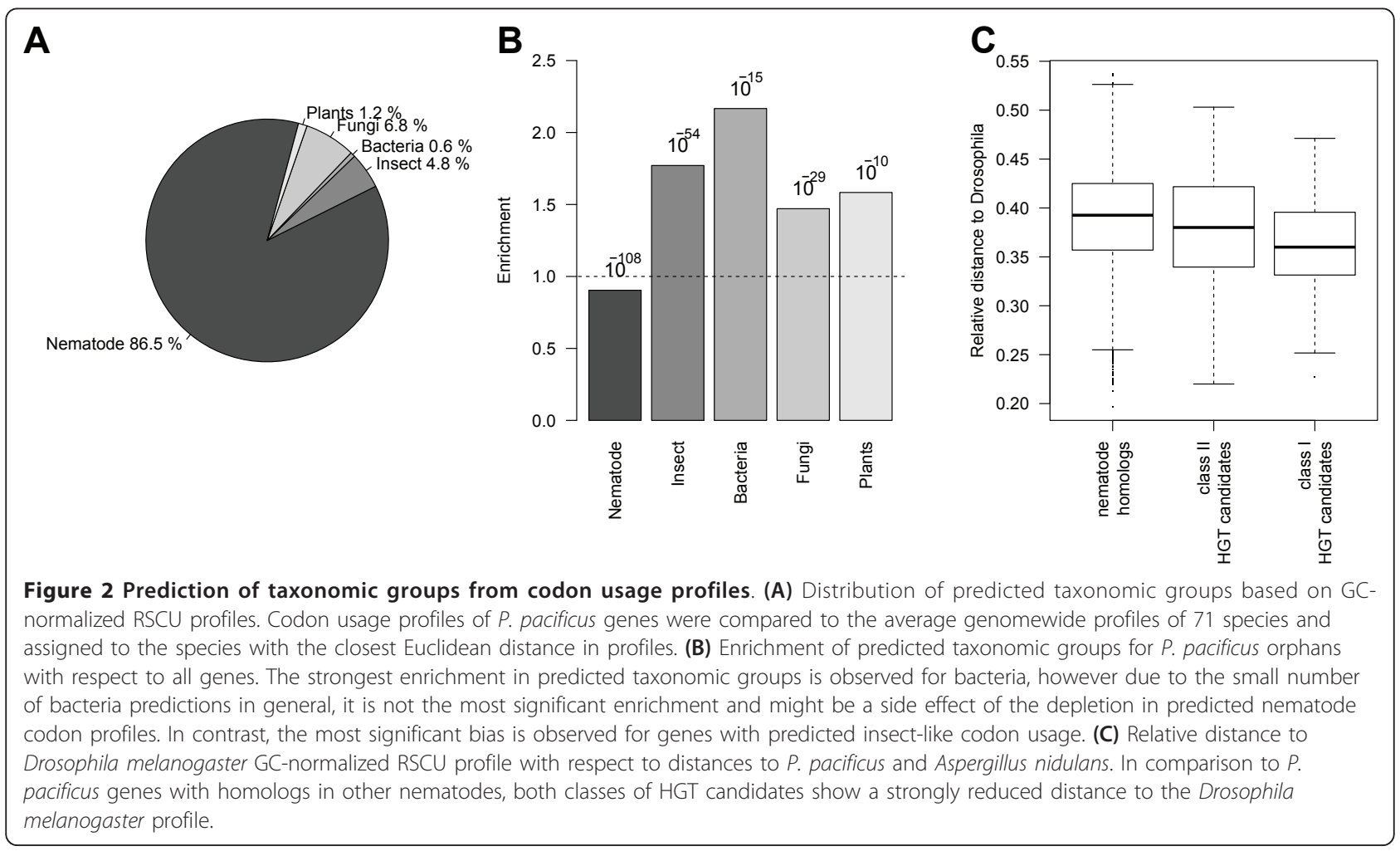


evidence of HGT from insects by comparing the results obtained from our codon usage analysis to HGT candidates defined by cross-species homology. We compiled a database including nematode as well as insect protein sequences to test whether HGT candidates defined by cross-species homology show a significant association with insect-like codon usage. We searched the blastp results for two classes of genes that would give rise to HGT candidates. First, we looked for P. pacificus orphan genes that had no homologous sequence in other nematodes but had a blastp hit $(e$-value $<0.001)$ in insects (class I). Second, we scanned for genes with homologs in other nematodes but with a minimum thousand fold smaller blastp $e$-value for a hit against any insect protein (class II). We identified 205 class I and 304 class II HGT candidates. The 509 HGT candidates with best blastp hits, taxonomic group predictions, and PFAM domains are presented in additional file 2 .

Most of the HGT candidates were predicted to be nematode genes based on their codon usage, i.e. $76.5 \%$ of class I and $84.7 \%$ of class II HGT candidates. This result indicates that the majority of genes acquired by HGT have been adapted to the codon usage of the host genome, an observation that has also been suggested by Karlin et al. [13]. Alternatively, this finding might be due to the similarity between the codon usage profiles of the donor and host genomes.

However, for the remaining HGT candidates, evidence for the taxonomic group of donors can be obtained. $9 \%$ of class II HGT candidates exhibit insect-like codon usage, which represents a significant 1.9 fold enrichment relative to the fraction of predicted insect-like codon usage among all genes $\left(P<10^{-5}\right.$, Fisher's exact test). For the 205 class I candidates there was no enrichment in insect-like codon usage, but a 1.3 fold enrichment in predicted fungal profiles (see Discussion) which is in contrast to the 9217 orphan genes (see above). To test whether both classes of HGT candidates show a higher similarity to insect-like codon usages than genes with nematode homologs, we considered relative rather than absolute distances of single genes to genomewide profiles of nematodes, fungi, and insects. In particular, we used the distance relative to the sum of nematode, fungal and insect distances, where Drosophila and Aspergillus represent insects and fungi, respectively. We found that both HGT candidate classes show a significant reduction in distance to the Drosophila profile $\left(P<10^{-13}\right.$ for class I candidates and $P<0.01$ for class II candidates, Wilcoxon test, Figure 2C).

These results suggest, that despite the close similarity between insect, fungal, and nematode codon usages, HGT candidates defined by cross-species homology show a significantly increased similarity to insect-like codon usages. Taken together, the genomewide computational analysis of HGT candidates defined by cross-species homology supports an association with insect-like codon usage.

\section{Phylogenetic analysis reveals integration of non-LTR retrotransposons}

To elucidate what genes could have been acquired by $P$. pacificus, we looked more closely into the gene sets identified by cross-species homologies. First, we used the previously identified Diapausin gene as a control [9]. Diapausins encode diapause-specific peptides that provide antifungal activity by acting as $\mathrm{Ca} 2+$ channel blockers [18]. Among the HGT candidates supported by crossspecies homologies and predicted insect-like codon usage, we indeed identified two genes that showed closest similarity to Diapausins from Spodoptera and Gastrophysa. The P. pacificus genome contains four other members of this gene family (PF08036.4) which are all among the HGT candidates defined by cross-species homology; Figure 3A shows a phylogenetic tree of this gene family. All P. pacificus Diapausin genes originate from a common sequence and show closest similarity to the leaf beetle Diapausin. This positive control confirms the effectiveness of our approach towards the identification of HGT candidates.

Table 2 shows the 20 most abundant PFAM domains that were found in the set of 509 HGT candidates defined by cross-species homology. The two most abundant domains correspond to a reverse transcriptase (PF00078.20) and endonuclease domain (PF03372.16). Both domains also occur in HGT candidates that are supported by cross-species homologies and predicted insect-like codon usage. These genes are remote homologs of the C. elegans retrotransposable element Rte-1 [19], a member of the non-LTR retrotransposable elements. Rte- 1 encodes an endonuclease and a reverse transcriptase enclosed by target site duplication sequences at the 5' and 3' end. Similar examples of a Rte-1-like HGT events have been reported from plants to fish and from arthropods to reptiles [20]. A more recent phylogenetic analysis observed a greater similarity of a P. pacificus reverse transcriptase to that of Bombyx mori sequences without any further discussion [21].

In total, the P. pacificus genome encodes 159 genes with a strong similarity to reverse transcriptase domains (e-value $<0.001) .70$ of these domains exhibit a higher similarity to a reverse transcriptase from insects than to the closest homolog within the nematode phylum. The remaining reverse transcriptase domains have closest homologs in other nematodes. Together, these findings indicate that the $P$. pacificus genome contains ancient homologs of the C. elegans Rte-1 elements as well as copies that might have originated from insects. Figure $3 \mathrm{~B}$ shows a phylogenetic tree of reverse transcriptase 


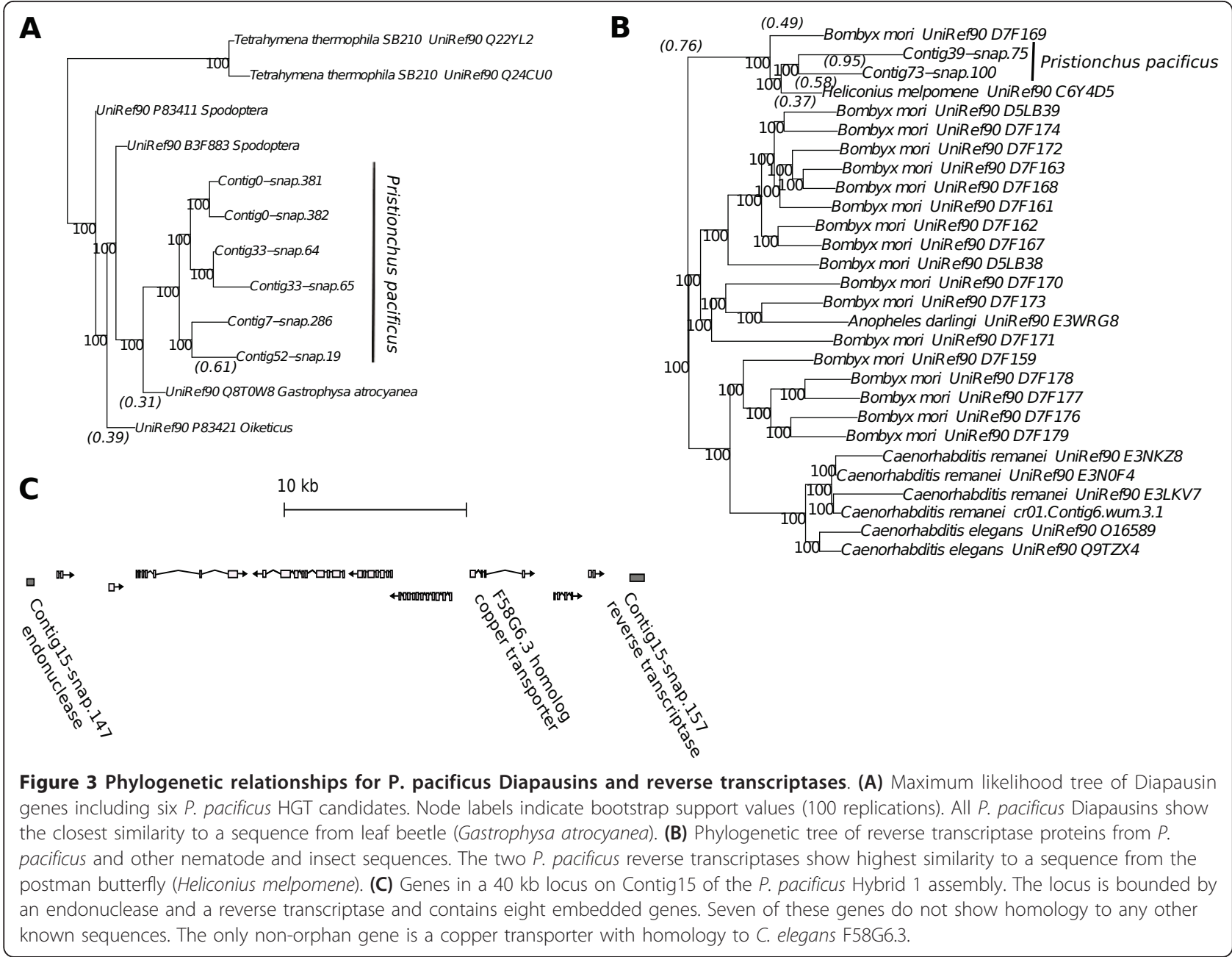

proteins including two $P$. pacificus genes with closest homologs in insects.

\section{Orphan genes and HGT candidates preferentially colocalize near retrotransposons}

The HGT candidates supported by cross-species homologies and predicted insect-like codon usage contain, in addition to the non-LTR retrotransposons, a second retrotransposon associated gene. This gene shows highest similarity to a gene prediction encoding a gag protein of a retrotransposon (PF03732.10, $e$-value $<10^{-16}$ ) from the red flower beetle Tribolium castaneum (see additional file 2). Gag proteins are known to mediate the telomerspecific transposition of retrotransposons for telomer maintenance in Drosophila [22].

The discovery of retrotransposons in HGT candidates led us to wonder whether retrotransposons are not only detectable outcomes of HGT events, but are possible mediators of HGT by cotransposition of intervening genes. A number of studies reported transfers of genetic material across species borders by means of molecular parasites. These reports include the transfer of $P$ elements between Drosophila species [23] and a transfer of a fungal endonuclease gene into an intron of the coxl gene in the plant Peperomia polybotrya [24] (see [25] for review).

Therefore, we searched for endonuclease and reverse transcriptase bounded intervals of up to $40 \mathrm{~kb}$ in the $P$. pacificus genome. We identified one prominent example that encompasses 10 genes, which are bounded by an endonuclease and a reverse transcriptase in the same orientation (Figure 3C). Out of the eight intervening genes, only one is found in other nematodes, whereas no homologous sequence could be detected for the remaining ones. This suggests that retrotransposons are able to carry more than just the genes required for transposon activity.

Next, we tested for a genomewide tendency of orphan genes to cluster in the vicinity of retrotransposons and searched for significant enrichment of orphan genes and class II HGT candidates in their vicinity. We first defined a reference set of all retrotransposon genes (reverse 
Table 2 Pfam domains in HGT candidates

\begin{tabular}{|c|c|c|c|c|}
\hline Accession ID & Description & $N$ & Enrichment & $p$-value \\
\hline PF00078.20 & Reverse transcriptase (RNA-dependent DNA polymerase) & 58 & $14.0 \times$ & $<10^{-49}$ \\
\hline PF03372.16 & Endonuclease/Exonuclease/phosphatase family & 27 & $18.8 \times$ & $<10^{-26}$ \\
\hline PF00096.19 & Zinc finger, $\mathrm{C} 2 \mathrm{H} 2$ type & 14 & $5.1 \times$ & $<10^{-5}$ \\
\hline PF00067.15 & Cytochrome P450 & 11 & $2.5 \times$ & $<0.01$ \\
\hline PF00069.18 & Protein kinase domain & 9 & $0.9 \times$ & 1 \\
\hline PF07714.10 & Protein tyrosine kinase & 9 & $1.0 \times$ & 0.9 \\
\hline PF00097.18 & Zinc finger, C3HC4 type (RING finger) & 8 & $3.1 \times$ & $<0.01$ \\
\hline PF00089.19 & Trypsin & 6 & $6.4 \times$ & $<0.01$ \\
\hline PF08036.4 & Diapausin family of antimicrobial peptide & 6 & $38.3 \times$ & $<10^{-8}$ \\
\hline PF08953.4 & Domain of unknown function (DUF1899) & 6 & $25.6 \times$ & $<10^{-6}$ \\
\hline PF00076.15 & RNA recognition motif (RRM) & 5 & $1.6 x$ & 0.3 \\
\hline PF00644.13 & Poly(ADP-ribose) polymerase catalytic domain & 5 & $24.0 \times$ & $<10^{-5}$ \\
\hline PF00856.21 & SET domain & 5 & $5.8 \times$ & $<0.01$ \\
\hline PF03732.10 & Retrotransposon gag protein & 5 & $24.0 \times$ & $<10^{-5}$ \\
\hline PF12171.1 & Zinc-finger double-stranded RNA-binding & 5 & $3.8 \times$ & $<0.05$ \\
\hline PF00092.21 & von Willebrand factor type A domain & 4 & $3.2 \times$ & 0.06 \\
\hline PF00106.18 & short chain dehydrogenase & 4 & $1.4 \times$ & 0.4 \\
\hline PF00178.15 & Ets-domain & 4 & $8.5 \times$ & $<0.01$ \\
\hline PF00005.20 & $\mathrm{ABC}$ transporter & 3 & $1.4 \times$ & 0.6 \\
\hline PF00098.16 & Zinc knuckle & 3 & $3.7 x$ & $<0.05$ \\
\hline
\end{tabular}

The 20 most abundant Pfam domains (e-value <0.001) in 509 HGT candidates defined by cross-species homology are shown. Enrichments factors and FDR corrected $p$-values were calculated relative to all $P$. pacificus genes with annotated PFAM domains.

transcriptases (PF00078.20), endonucleases (PF03372.16), and gag proteins (PF03732.10)) with higher similarity to insects than to nematodes. We then repeatedly sampled non-overlapping genomic locations of equal number and size distribution as that of the merged $30 \mathrm{~kb}$ upstream and downstream regions of the retrotransposon genes. In total, these regions span 5.2 Mb and encompass 352 orphan genes and class II HGT candidates, representing a $1.15 \pm$ 0.08 fold enrichment relative to randomly sampled genomic locations ( $P=0.034,1000$ iterations). Thus, HGT candidates nonetheless show a tendency to cluster around retrotransposon genes. However, we could not detect any significant trend towards either upstream or downstream regions of reverse transcriptases and endonucleases. These findings are in agreement with the assumption that insect retrotransposons might have been used as vectors for integration of foreign DNA into the $P$. pacificus genome.

\section{HGT candidates defined by cross-species homology are conserved in the genus Pristionchus}

An association with retrotransposons suggests that more or less random genetic material has been integrated into the $P$. pacificus genome. For evolutionary significance of HGT events, integration into the host biology and permanence are required [26]. Even if foreign DNA can be integrated into a host genome, it will be rapidly degraded by neutral evolution, unless it will prove beneficial for the host. To test, whether the HGT candidates have been introduced into the $P$. pacificus genome only recently, we searched for homologous sequences in the low coverage genomes of Pristionchus entomophagus and Pristionchus maupasi (blastn $e$-value <0.001) [9]. We found homologous sequences for 218 (41.6\%) of HGT candidates in at least one other Pristionchus species, 105 (20.6\%) of HGT candidates showed evidence for conservation in both species, indicating that a substantial fraction of HGT candidates date back to ancestral sequences within the genus Pristionchus. However, more data will be needed to evaluate whether these genes evolve neutrally or are under any kind of evolutionary constraint.

\section{Discussion}

Novel sequencing technologies have dramatically increased the number of available genome sequences over the last few years. However, the biological value of a new genome sequence is limited due to the lack of knowledge about homologous sequences in other organisms. The absence of homology to any known sequence, as in the case for a large fraction of $P$. pacificus genes $[9,10]$, exemplifies our lack of knowledge about the genome content of this model organism. Therefore, we have started to complement the available $P$. pacificus genome, transcriptome and proteome data with next generation sequencing approaches of related species [3,9]. This approach has facilitated a mechanistic understanding of some of the 
HGT events that occurred in the evolutionary lineage giving rise to $P$. pacificus. For cellulase genes acquired from microbes, $P$. pacificus and related Pristionchus nematodes show functional assimilation, high gene turnover and rapid sequence diversification associated with positive selection [3].

In $P$. pacificus, the scarab beetle-associated ecology might result in a number of potential donors for HGT. The decaying beetle is an ecosystem consisting of bacteria, fungi, nematodes and presumably, a large number of unicellular eukaryotes. The previously described examples of cellulase and Diapausin genes clearly indicate that microbes and insects, at least, must be considered as potential HGT donors into the P. pacificus genome. One inroad into the identification of HGT events is a computational archaeology approach as originally described for E. coli [1].

In this study we have hypothesized that a substantial fraction of the $P$. pacificus orphans might be introduced into the genome by means of HGT. Hereby we refer to an orphan gene as a gene with no similarity to any other nematode sequence. Under the assumption that some horizontally transferred genes may exhibit a codon usage bias that is more similar to the donor genome than to the acceptor genome $[1,13]$, we could show that a fraction of $P$. pacificus orphans exhibits an atypical codon usage relative to the rest of the genome. The fact that the majority of orphan genes show a codon usage typical for nematodes might be due to two circumstances. First, HGT events most likely occurred repeatedly with more recent HGT events preferentially showing a codon usage bias. Second, with multiple potential donors, no common patterns of atypical codon usage are expected. For example, nematodes, insects and fungi show closely related codon usage patterns, whereas protozoans and other microbes, all of which are potential donors for HGT, exhibit very different codon usages. In our analysis, we found a similarity in codon usages for insects, nematodes and fungi. GC-normalized RSCU distances of P. pacificus genes to the genomewide profiles of $P$. pacificus, Drosophila melanogaster, and Aspergillus nidulans showed strong correlations $(r>0.87$, Pearson). This circumstance highlights the need for a careful investigation of potential HGT events. We consider the work presented in this study as a novel computational entry road towards the identification of HGT patterns in P. pacificus.

In addition to the strong association of orphan genes with atypical codon usage, we could characterize this codon usage pattern by comparison to genomewide profiles for 71 species corresponding to six taxonomic groups. The extent to which codon usage profiles can predict species and taxonomic groups is still limited. However, comparisons of subsets of genes against all genes may help uncover the domain or phylum, from which these genes entered the $P$. pacificus genome. The most significant enrichment was detected for insect-like codon usage $\left(P<10^{-54}\right)$.

It is important to note that atypical patterns of codon usage may also arise from other sources such as translational efficiency or secondary structures (see [15] for review). Thus analysis of codon usage alone may not be sufficient to support the proposed HGT events. We therefore complemented this analysis by cross-species comparisons to identify genes that show greatest similarity to homologs in insects.

We identified 509 HGT candidates using homology searches against a combined nematode and insect protein database and scanning for genes bearing greater resemblance to insect genes than to the closest homologs within the nematode phylum. These HGT candidates showed a significantly higher similarity to insect-like codon usage profiles. Further investigations revealed that in addition to the previously identified Diapausins (Table 2) [9], many of these genes encode endonuclease and reverse transcriptase proteins. Since 70 of the 159 P. pacificus reverse transcriptase sequences show a higher degree of similarity to those of insects, we speculate that reintroduction of these elements from insects represents one mechanism by which P. pacificus has acquired genes. Phylogenetic analysis of all HGT candidates identified by cross-species homology could provide more detailed information and further support for the proposed HGT events. Although P. pacificus is not an insect parasitic nematode, dauer larvae of $P$. pacificus are in constant physical contact with beetles [27]. After the death of the beetle, nematodes resume development and feed on microorganisms growing on the carcass, presumably for several generations [28]. Close physical contact between donor and recipient has been proposed as one criteria for HGT [5], making beetles a plausible candidate for HGT donors. While our data suggests that a substantial fraction of P. pacificus orphans originates from insect genomes, it is possible that HGT involves vectors as intermediate carriers. It is known that many viruses coexist with insects often in a species-specific interaction, so viruses are obvious candidates for HGT into $P$. pacificus. This hypothesis is supported by the finding that parts of the Diapausin genes found in leaf beetles and P. pacificus have also been observed in iridoviruses [18]. We therefore hypothesize that viruses are potential intermediate carriers that promote HGT events from insects into $P$. pacificus.

Our data however, strongly support a second scenario. We identified a large number of non-LTR retrotransposon sequences in the $P$. pacificus genome that have highest sequence similarity to insects. In addition to permanence, integration into the host's biology is one necessary features of HGT [26]. The non-LTR retrotransposons are unlikely to have a beneficial effect on the biology of $P$. pacificus. Thus the strong enrichment of 
retrotransposon associated genes among HGT candidates defined by cross-species homology seems counterintuitive. One explanation for this observation is that retrotransposons might have served as carriers of foreign genetic material into the $P$. pacificus genome. This hypothesis is supported by the fact that we detected a tendency for orphan genes and other HGT candidates to be colocalized near retrotransposon genes. It could provide one possible explanation for the presence of foreign retrotransposons and could serve as a model for how other genes might have integrated into the $P$. pacificus genome. An open question is the permanence of the transferred genetic material. Comparison with other Pristionchus species indicates, that a substantial fraction of HGT candidate dates back to ancestral Pristionchus sequences. However, more data from wild isolates will be needed to robustly measure the amount of selection acting on these genes.

\section{Conclusions}

The computational archaeology of the $P$. pacificus genome combines analysis of codon usage bias with phylogenetic analysis, both of which reveal evidence of HGT events from insects. We identified colocalized gene clusters surrounded by non-LTR retrotransposons, suggesting a mechanism of HGT that involves transposable elements $[23,24]$. This study highlights that multiple computational approaches are necessary to obtain an overview of HGT and other potential genomewide mechanisms contributing to the evolution of eukaryotic genomes.

\section{Methods}

\section{Identification of orphan genes}

We used the gene predictions for 24,231 Pristionchus pacificus genes [10] and downloaded gene annotations for 30,163 Caenorhabditis elegans transcripts (WS220), 21,991 Caenorhabditis briggsae transcripts (WS223), and 21,332 from Brugia malayi transcripts (WS222).

We compiled a protein sequence database for all nonPristionchus nematode protein sequences which included 20,359 Meloidogyne incognita proteins obtained from the $M$. incognita resources website [29], 157,761 protein translations of non-P. pacificus nematode ESTs from nematode.net [30] and non-Pristionchus nematode sequences from the UniRef90 database. We then searched this database for $P$. pacificus homologs using NCBI blastp (version 2.2.22) [31].

For 15,014 (62.0\%) of $P$. pacificus genes, at least one nematode homolog with $e$-value $<0.001$ could be identified. This translates to 9217 (38.0\%) orphan genes.

\section{GC content and codon usage profiles}

To eliminate biases due to recent gene expansions or alternatively spliced transcripts in the genomewide calculation of RSCU profiles, we clustered all transcripts with $\geq 80 \%$ sequence identity using cd-hit-est [32] (version 4.3 with -c 0.8 options).

We further restricted the analysis of codon usage to only those transcripts with at least 100 codons. This reduced the number of $P$. pacificus transcripts to 19,515 , 6370 of which are in the set of orphan genes. For each transcript with at least 100 codons, we determined the GC3 content for the whole gene and then calculated the GC-normalized RSCU values for a codon $x$ as

$$
\operatorname{RSCU}_{G C}(x)=\frac{f_{o b}(x)}{f_{\exp }(x)},
$$

where $f_{o b}(x)$ denotes the observed frequency of codon $x$ among all synonymous codons and $f_{\exp }(x)$ is calculated as

$$
f_{\text {exp }}(x)=\frac{p(x \mid G C 3)}{\sum_{y \in \operatorname{syn}(x)} p(y \mid G C 3)},
$$

where $\operatorname{syn}(x)$ denotes the set of all synonymous codons for $x$ and $p(x \mid G C 3)$ indicates the probability of codon $x$ for a given GC3 content. $p(x \mid G C 3)$ is calculated as the product of the three individual nucleotide probabilities given the GC3 content

$$
p(x \mid G C 3)=G C 3^{k} \times(1-G C 3)^{3-k},
$$

whereby $k$ denotes the number of nucleotides that are either $\mathrm{G}$ or $\mathrm{C}$. Deviation in RSCU values for single genes was computed as the Euclidean distance to the average genomewide RSCU profile. Analogously deviation in GC3 for a single gene was calculated as the absolute difference from the genomewide average GC3 content.

\section{Transcriptome and EST data}

We downloaded protein coding transcript annotations for 15 bacterial and 12 fungal genomes from Ensembl Bacterial and Fungal Mart (Release 8), and unique representative EST sequences from UniGene for 5 plant, 8 insect, and 1 amoebozoa species. These representative sequences were defined as the sequence with the longest region of high-quality sequence data for each UniGene EST cluster. In addition we downloaded nematode consensus EST sequences from nematode.net [30,33].

For each EST sequence we identified the coding sequence (CDS) as the longest sequence with no stop codons among all 6 potential reading frames; for that sequence, the transcript could start either at the first codon or at the last ATG. If the longest sequence was at least $300 \mathrm{bp}$ long and the second longest was at most $150 \mathrm{bp}$ long, we used this CDS for further analysis.

We obtained 17,189 transcripts for red harvester ant Pogonomyrmex barbatus (Gene Set v1.2) from the 
Hymenoptera Genome Database [34], 21,899 Drosophila melanogaster transcripts from the Ensembl 61 database, 14,623 transcripts for silkworm Bombyx mori from SilkDB [35], 11,062 transcripts for honey bee Apis mellifera (PreRelease2 OGS) from BeeBase [34], and 18,249 sequences for red flour beetle Tribolium castaneum from the Tribolium castaneum Genome Project website (Tcas2.0) [36].

\section{Prediction of species and taxonomic groups based on RSCU profiles}

To predict species given the GC-normalized RSCU values of a single gene, we compared the RSCU profile of the gene with genomewide profiles for all species and assigned it to the nearest neighbor, which we defined as the species with the closest Euclidean distance between RSCU profiles.

The accuracy of the nearest neighbor method for species and taxonomic group predictions was calculated as the fraction of correct predictions among all predictions. The reported accuracies are based on ten evaluations of data sets for 71 species (13 insects, 15 bacteria, 25 nematodes, 12 , fungi, 5 plants and 1 amoebozoa) with more than 1000 sequences containing greater than 100 codons. From each species 333 sequences were randomly drawn and put into the validation set.

\section{Identification of HGT candidates by cross-species homology and phylogenetic analysis}

To define HGT candidates we combined the nematode proteins with protein translations for the five insect genomes and insect and arthropod sequences from the UniRef90 data base. We searched for class I and II HGT candidates in the resulting set of 721,041 protein sequences using blastp.

For the non-LTR retrotransposon reverse transcriptase we used the program hmmsearch from the HMMer package (version 3.0) to scan the nematode and insect protein sequences for presence of a reverse transcriptase (1239 PF00078.20 hits with $e$-value $<10^{-10}$ ) domain. This set was extended by eight $P$. pacificus reverse transcriptases supported by cross-species homologies and predicted insect-like codon usage. To reduce the number of reverse transcriptase protein sequences that were used for the multiple alignment, we clustered the sequences with cdhit (60\% identity cutoff) and Transclust [37]. Only one cluster contained P. pacificus HGT candidates and other reverse transcriptases. We then used muscle (version 3.8.31, [38]) to align sequences and the phangorn $\mathrm{R}$ package [39] to estimate a maximum-likelihood tree under the LG amino acid substitution model [40] with the Gamma + I model to account for rate variation across sites [41]. For Diapausins we scanned the whole UniRef90 database for Diapausin domains (six PF08036.4 hits with $e$-value $<10^{-3}$ ). This set was extended by six $P$. pacificus Diapausins and subsequently used for alignment and maximum-likelihood tree estimation.

\section{Additional material}

\begin{abstract}
Additional file 1: Distribution of GC-normalized RSCU deviation from the genomewide profile. Histogram of Euclidean distances between GC-normalized RSCU values of single genes and the genomewide profile of $P$. pacificus. For all orphans and non orphans, the frequency of genes in each Euclidean distance bin is shown.

Additional file 2: List of HGT candidates defined by cross-species homology. List of HGT candidates with best blastp hit, predicted taxonomic group based on codon usage, and identified PFAM domains.
\end{abstract}

\section{Acknowledgements}

We thank R. I. Molnar for helpful advice in the phylogenetic analysis and A. Streit and $\mathrm{P}$. Basu for critically reading the manuscript. This project was funded by the Max-Planck Society.

\section{Authors' contributions}

CR and RJS conceived the work. CR analyzed the data. CR and RJS wrote the paper. Both authors read and approved the final manuscript.

Received: 14 June 2011 Accepted: 15 August 2011

Published: 15 August 2011

\section{References}

1. Lawrence JG, Ochman H: Molecular archaeology of the Escherichia coli genome. Proc Natl Acad Sci USA 1998, 95(16):9413-9417.

2. Boucher Y, Douady CJ, Papke RT, Walsh DA, Boudreau MER, Nesbø CL, Case RJ, Doolittle WF: Lateral gene transfer and the origins of prokaryotic groups. Annu Rev Genet 2003, 37:283-328.

3. Mayer WE, Schuster LN, Bartelmes G, Dieterich C, Sommer RJ: Horizontal gene transfer of microbial cellulases into nematode genomes is associated with functional assimilation and gene turnover. BMC Evol Biol 2011, 11:13.

4. Danchin EGJ, Rosso MN, Vieira P, de Almeida-Engler J, Coutinho PM, Henrissat B, Abad P: Multiple lateral gene transfers and duplications have promoted plant parasitism ability in nematodes. Proc Natl Acad Sci USA 2010, 107(41):17651-17656.

5. Mitreva M, Smant G, Helder J: Role of horizontal gene transfer in the evolution of plant parasitism among nematodes. Methods Mol Biol 2009, 532:517-535.

6. Hotopp JCD, Clark ME, Oliveira DCSG, Foster JM, Fischer P, Torres MCM, Giebel JD, Kumar N, Ishmael N, Wang S, Ingram J, Nene RV, Shepard J, Tomkins J, Richards S, Spiro DJ, Ghedin E, Slatko BE, Tettelin H, Werren JH: Widespread lateral gene transfer from intracellular bacteria to multicellular eukaryotes. Science 2007, 317(5845):1753-1756.

7. Sommer RJ: Evolution of development in nematodes related to $C$. elegans. WormBook 2005, 1-17.

8. Herrmann M, Mayer WE, Sommer RJ: Nematodes of the genus Pristionchus are closely associated with scarab beetles and the Colorado potato beetle in Western Europe. Zoology (Jena) 2006, 109(2):96-108

9. Dieterich C, Clifton SW, Schuster LN, Chinwalla A, Delehaunty K, Dinkelacker I, Fulton L, Fulton R, Godfrey J, Minx P, Mitreva M, Roeseler W, Tian H, Witte H, Yang SP, Wilson RK, Sommer RJ: The Pristionchus pacificus genome provides a unique perspective on nematode lifestyle and parasitism. Nat Genet 2008, 40(10):1193-1198.

10. Borchert N, Dieterich C, Krug K, Schütz W, Jung S, Nordheim A, Sommer RJ, Macek B: Proteogenomics of Pristionchus pacificus reveals distinct proteome structure of nematode models. Genome Res 2010, 20(6):837-846

11. Karlin S: Global dinucleotide signatures and analysis of genomic heterogeneity. Curr Opin Microbiol 1998, 1(5):598-610. 
12. Arvey AJ, Azad RK, Raval A, Lawrence JG: Detection of genomic islands via segmental genome heterogeneity. Nucleic Acids Res 2009, 37(16):5255-5266

13. Karlin S, Mrázek J, Campbell AM: Codon usages in different gene classes of the Escherichia coli genome. Mol Microbiol 1998, 29(6):1341-1355.

14. Merkl R: SIGI: score-based identification of genomic islands. BMC Bioinformatics 2004, 5:22.

15. Plotkin JB, Kudla G: Synonymous but not the same: the causes and consequences of codon bias. Nat Rev Genet 2011, 12:32-42.

16. Cutter AD, Wasmuth JD, Blaxter ML: The evolution of biased codon and amino acid usage in nematode genomes. Mol Biol Evol 2006, 23(12):2303-2315.

17. Breiman L: Random Forests. Mach Learn 2001, 45:5-32.

18. Tanaka H, Sato K, Saito Y, Yamashita T, Agoh M, Okunishi J, Tachikawa E, Suzuki K: Insect diapause-specific peptide from the leaf beetle has consensus with a putative iridovirus peptide. Peptides 2003 24(9):1327-1333.

19. Youngman $\mathrm{S}$, van Luenen $\mathrm{HG}$, Plasterk $\mathrm{RH}$ : Rte-1, a retrotransposon-like element in Caenorhabditis elegans. FEBS Lett 1996, 380(12):1-7.

20. Zupunski V, Gubensek F, Kordis D: Evolutionary dynamics and evolutionary history in the RTE clade of non-LTR retrotransposons. Mol Biol Evol 2001, 18(10):1849-1863.

21. Tay WT, Behere GT, Batterham P, Heckel DG: Generation of microsatellite repeat families by RTE retrotransposons in lepidopteran genomes. BMC Evol Biol 2010, 10:144.

22. Rashkova S, Karam SE, Kellum R, Pardue ML: Gag proteins of the two Drosophila telomeric retrotransposons are targeted to chromosome ends. J Cell Biol 2002, 159(3):397-402.

23. Daniels SB, Peterson KR, Strausbaugh LD, Kidwell MG, Chovnick A: Evidence for horizontal transmission of the $P$ transposable element between Drosophila species. Genetics 1990, 124(2):339-355.

24. Vaughn JC, Mason MT, Sper-Whitis GL, Kuhlman P, Palmer JD: Fungal origin by horizontal transfer of a plant mitochondrial group I intron in the chimeric Coxl gene of Peperomia. J Mol Evol 1995, 41(5):563-572.

25. Schaack S, Gilbert C, Feschotte C: Promiscuous DNA: horizontal transfer of transposable elements and why it matters for eukaryotic evolution. Trends Ecol Evol 2010, 25(9):537-546.

26. Blaxter M: Symbiont genes in host genomes: fragments with a future? Cell Host Microbe 2007, 2(4):211-213

27. Weller AM, Mayer WE, Rae R, Sommer RJ: Quantitative assessment of the nematode fauna present on Geotrupes dung beetles reveals species-rich communities with a heterogeneous distribution. J Parasitol 2010, 96(3):525-531.

28. Mayer MG, Sommer RJ: Natural variation in Pristionchus pacificus dauer formation reveals cross-preference rather than self-preference of nematode dauer pheromones. Proc Biol Sci 2011.

29. Abad P, Gouzy J, Aury JM, Castagnone-Sereno P, Deleury E, PerfusBarbeoch L, Anthouard V, Artiguenave F, Blok VC, Caillaud MC, et al: Genome sequence of the metazoan plant-parasitic nematode Meloidogyne incognita. Nat Biotechnol 2008, 26(8):909-915.

30. Martin J, Abubucker S, Wylie T, Yin Y, Wang Z, Mitreva M: Nematode.net update 2008: improvements enabling more efficient data mining and comparative nematode genomics. Nucleic Acids Res 2009, , 37 Database: D571-D578.

31. Altschul SF, Gish W, Miller W, Myers EW, Lipman DJ: Basic local alignment search tool. J Mol Biol 1990, 215(3):403-410.

32. Li W, Godzik A: Cd-hit: a fast program for clustering and comparing large sets of protein or nucleotide sequences. Bioinformatics 2006 , 22(13):1658-1659.

33. Parkinson J, Mitreva M, Whitton C, Thomson M, Daub J, Martin J, Schmid R, Hall N, Barrell B, Waterston RH, McCarter JP, Blaxter ML: A transcriptomic analysis of the phylum Nematoda. Nat Genet 2004, 36(12):1259-1267.

34. Munoz-Torres MC, Reese JT, Childers CP, Bennett AK, Sundaram JP, Childs KL, Anzola JM, Milshina N, Elsik CG: Hymenoptera Genome Database: integrated community resources for insect species of the order Hymenoptera. Nucleic Acids Res 2011, , 39 Database: D658-D662.

35. Duan J, Li R, Cheng D, Fan W, Zha X, Cheng T, Wu Y, Wang J, Mita K, Xiang Z, Xia Q: SilkDB v2.0: a platform for silkworm (Bombyx mori) genome biology. Nucleic Acids Res 2010, 38 Database: D453-D456.

36. Consortium TGS: The genome of the model beetle and pest Tribolium castaneum. Nature 2008, 452(7190):949-955.
37. Wittkop T, Emig D, Truss A, Albrecht M, Böcker S, Baumbach J: Comprehensive cluster analysis with Transitivity Clustering. Nat Protoc 2011, 6(3):285-295

38. Edgar RC: MUSCLE: multiple sequence alignment with high accuracy and high throughput. Nucleic Acids Res 2004, 32(5):1792-1797.

39. Schliep KP: phangorn: phylogenetic analysis in R. Bioinformatics 2011, 27(4):592-593.

40. Le SQ, Gascuel O: An improved general amino acid replacement matrix. Mol Biol Evol 2008, 25(7):1307-1320.

41. Yang Z: Maximum likelihood phylogenetic estimation from DNA sequences with variable rates over sites: approximate methods. J Mol Evol 1994, 39(3):306-314.

doi:10.1186/1471-2148-11-239

Cite this article as: Rödelsperger and Sommer: Computational archaeology of the Pristionchus pacificus genome reveals evidence of horizontal gene transfers from insects. BMC Evolutionary Biology 2011 $11: 239$.

\section{Submit your next manuscript to BioMed Central and take full advantage of:}

- Convenient online submission

- Thorough peer review

- No space constraints or color figure charges

- Immediate publication on acceptance

- Inclusion in PubMed, CAS, Scopus and Google Scholar

- Research which is freely available for redistribution

Submit your manuscript at www.biomedcentral.com/submit
C) Biomed Central 